\title{
电沉积表面修饰对染料敏化太阳电池微观性能影响机理研究
}

\author{
寇东星 $^{a} \quad$ 刘伟庆 $^{a, b} \quad$ 胡林华 $^{a}$ 陈双宏 ${ }^{a}$ 黄阳 $^{a}$ 戴松元 $^{*}, a$ \\ $\left({ }^{a}\right.$ 中国科学院新型薄膜太阳电池重点实验室 中国科学院等离子体物理研究所 合肥 230031) \\ ( ${ }^{b}$ 南昌航空大学测试与光电工程学院无损检测技术教育部重点实验室＼cjkstart南昌 330063)
}

\begin{abstract}
摘要 为了改善染料敏化太阳电池内电子的传输复合过程, 研究者尝试不同方法制备或改性 $\mathrm{TiO}_{2}$ 薄膜. 对 $\mathrm{TiO}_{2}$ 薄膜 进行后处理, 在其表面引入一层小颗粒层, 是一种有效的方法并被广泛研究. 通过对 $\mathrm{TiO}_{2}$ 薄膜不同时间的电沉积表面 修饰, 细致研究了表面修饰后染料敏化太阳电池微观性能的变化机制. 采用阳极氧化法在 $\mathrm{TiCl}_{3}$ 水溶液中对 $\mathrm{TiO}_{2}$ 薄膜 进行电沉积后处理, 将溶液 $\mathrm{pH}$ 值调至 2.2 , 装置的反应速率由恒电位仪控制. 不同沉积时间电池带边移动以及电子传 输复合的动力学过程, 借助强度调制光电流谱(IMPS)/强度调制光电压谱(IMVS)和电化学阻抗谱(EIS)等探测技术表征. 研究表明, 电沉积在 $\mathrm{TiO}_{2}$ 薄膜表面引入了大量浅能级陷阱态, 以致电势较高时电容随沉积时间延长增加明显. 不同时 间的电沉积表面修饰在 $\mathrm{TiO}_{2}$ 薄膜表面形成了新的小颗粒层并改善了 $\mathrm{TiO}_{2}$ 颗粒间接触，在改善电子注入及收集过程的 同时, 也有效抑制了内部电子复合. IMPS/IMVS 结果表明, 电沉积对动力学过程改善的效果受光强影响明显, 弱光下 作用更为突出. 此外, 电池开路电压主要受带边移动及内部复合变化影响, 随沉积时间延长, 表面电荷的增多使 $\mathrm{TiO}_{2}$ 薄膜带边逐渐正移，有效改善了光电流却限制了开路电压的提升. 在适合的电沉积时间下，电沉积表面修饰可以同时 改善光电流和光电压.
\end{abstract}

关键词＼cjkstart染料敏化; 太阳电池；强度调制光电流谱/光电压谱; 电子复合; 电沉积

\section{Influence of Electrodeposition Post-Treatment on Microcosmic Performances in Dye-sensitized Solar Cells}

\author{
Kou, Dongxing ${ }^{a} \quad$ Liu, Weiqing ${ }^{a, b}$ \\ Huang, Yang ${ }^{a}$ \\ Hu, Linhua ${ }^{a}$ \\ Dai, Songyuan*,a \\ Chen, Shuanghong ${ }^{a}$
}

$\left({ }^{a}\right.$ Key Laboratory of Novel Thin Film Solar Cells, Institute of Plasma Physics, Chinese Academy of Sciences, Hefei 230031)

$\left({ }^{b}\right.$ Key Laboratory of Nondestructive Testing of Ministry of Education, School of Measuring and Optical Engineering, Nanchang Hangkong University, Nanchang 330063)

\begin{abstract}
Over the last decade, different techniques had been employed to prepare nanoporous $\mathrm{TiO}_{2}$ electrode, aiming to improve the electron transport and depress electron recombination processes in dye-sensitized solar cells (DSCs). An effective method was a post-treatment of the $\mathrm{TiO}_{2}$ films in which an extra layer of $\mathrm{TiO}_{2}$ was grown onto the $\mathrm{TiO}_{2}$ nanoparticles constituting the films. Different explanations of the working principle of this coating had been reported. In the present work, the influence of surface modification of $\mathrm{TiO}_{2}$ films on microcosmic performances of DSCs was investigated. Research was developed by the post-treatment of $\mathrm{TiO}_{2}$ films using electrodeposition method. $\mathrm{Ti}^{4+}$ oxide films were deposited on $\mathrm{TiO}_{2}$ electrode by anodic oxidative hydrolysis of acidic aqueous $\mathrm{TiCl}_{3}$ solutions. The $\mathrm{pH}$ was adjusted to 2.2 and the rate of oxidation of $\mathrm{Ti}^{3+}$ at the anode was controlled by an almost constant anodic current using constant potential rectifier. The processes of electron recombination, transport and band edge movement were detected by intensity-modulated photocurrent spectroscopy (IMPS)/intensity-modulated photovoltage spectroscopy (IMVS) and electrochemical impedance spectroscopy (EIS). It was found that the capacitance of $\mathrm{TiO}_{2}$ electrode evidently increased with increasing electrodeposition time at high potentials due to the risen density of shallow traps. As the formation of small particles on the surface of $\mathrm{TiO}_{2}$ films and the enhancement of particle connections, the post-treatment with different electrodeposition time improved the charge injection and transport processes and depressed the electron recombination effectively. IMPS/IMVS measurements indicated that the effects of surface modification almost relied on light intensities, which would perform more prominent function at low intensities. Additionally, the open-circuit photovoltage $\left(V_{\mathrm{oc}}\right)$ was found to be mainly effected by the conduction band edge shift and the variation of charge recombination process. With increasing electrodeposition time, the conduction band was ordinally positive
\end{abstract}

\footnotetext{
*E-mail: sydai@ipp.ac.cn

Received January 6, 2013; published April 3, 2013.
}

Project supported by the National Basic Research Program of China (Grant No. 2011CBA00700), the National High Technology Research and Development Program of China (Grant No. 2011AA050527), the National Natural Science Foundation of China (Grant No. 21003130, 61204075 and 21173228), Funds of the Hefei Institutes of Physical Science in Innovation Engineering (Grant No. Y05FCQ1125) and the China Postdoctoral Science Foundation (Grant Nos. 20110490835, 2012T50581).

项目受国家重点基础研究发展计划(No. 2011CBA00700)、国家高技术研究发展计划(No. 2011AA050527)、国家自然科学基金(Nos. 21003130, 61204075, 21173228)、中国博士后科学基金(Nos. 20110490835, 2012T50581)和合肥物质科学研究院创新项目(No. Y05FCQ1125)资助. 
shift due to the increase of surface charge, which contributed an improvement of short-circuit current $\left(J_{\mathrm{sc}}\right)$ but a limitation of $V_{\text {oc }}$. Overall, an optimal modification time was needed to achieve a higher $J_{\text {sc }}$ and $V_{\text {oc }}$.

Keywords dye-sensitized; solar cells; IMPS/IMVS; electron recombination; electrodeposition

\section{1 引言}

近年来基于能源危机的加深及传统硅电池的成本 技术等问题, 开展新型太阳电池的研究成为必然 ${ }^{[1 \sim 3]}$. 瑞士联邦理工学院(洛桑)Grätzel 组自 1991 年将纳米 $\mathrm{TiO}_{2}$ 多孔薄膜电极替代染料敏化太阳电池(DSC)中传统 的单晶 $\mathrm{TiO}_{2}$ 电极并取得突破性进展以来 ${ }^{[4]}$, 过去 20 年 一直引领高效 DSC 的发展, 目前最高效率达到 $12.3 \%{ }^{[5]}$, 展现了良好的商业应用前景. DSC 环境友好、制作成本 低且工艺简单, 主要由染料敏化的 $\mathrm{TiO}_{2}$ 光阳极、电解质 和对电极三部分组成 ${ }^{[6,7]}$.

当 DSC 受到太阳光照射时, 染料分子吸收能量由 基态跃迁为激发态, 产生的电子空穴对在 Dyed- $\mathrm{TiO}_{2}$ /电 解液界面分离, 电子注入 $\mathrm{TiO}_{2}$ 导带, 经膜内传输至导电 玻璃基底收集并经由外电路流向对电极, 形成光电流的 循环 ${ }^{[8]}$. 通常半导体与电解液接触时, 其界面处会形成 空间电荷区(耗尽层)以平衡费米能级及离子间相互作用, 耗尽层中形成的电场可以使光生电荷在界面处发生有 效分离. 但是, 传统多孔 $\mathrm{TiO}_{2}$ 薄膜颗粒尺寸仅 $20 \sim 30$ $\mathrm{nm}$, 不足以支持空间电荷区的形成, 因此 DSC 实际工 作时 Dyed- $\mathrm{TiO}_{2}$ /电解液界面电子与 $\mathrm{I}_{3}^{-}$复合非常严重 ${ }^{[9]}$.

研究者尝试多种表面修饰方法对 DSC 性能进行改 善, 采用 $\mathrm{TiCl}_{4}$ 溶液、溶胶和电沉积等能够很好地加快 多孔膜内电子传输或抑制界面处电子复合 ${ }^{[1,10 ~ 12] . ~}$. 研究 报道, $\mathrm{TiCl}_{4}$ 溶液、电沉积的处理效果主要受 $\mathrm{TiO}_{2}$ 薄膜本 体材料、溶液浓度及处理时间影响 ${ }^{[13]}$. 大量实验及机理 研究表明, 表面修饰在 $\mathrm{TiO}_{2}$ 薄膜表面新生成的小颗粒 层, 可以有效增加薄膜表面积, 改善多孔薄膜纳米颗粒 间的接触 ${ }^{[9,12,14 \sim 16]}$ 并抑制电子与 $\mathrm{I}_{3}^{-}$间的复合. 上述动力 学过程的变化虽然使光电流得到明显改善, 但研究对于 光电压的变化却没有统一结论 ${ }^{[13,14,16]}$. Lee 等 ${ }^{[13]}$ 通过改 变 $\mathrm{TiCl}_{4}$ 溶液浓度的电池性能研究发现, 表面积的增大 使开路电压有所降低; 而 $\mathrm{Xu}$ 等 ${ }^{[17]}$ 采用电沉积处理 $\mathrm{TiO}_{2}$ 多孔薄膜发现, 表面修饰很好的抑制了电子复合, 提高 了开路电压.

目前相关 $\mathrm{TiCl}_{4}$ 、电沉积等报道大多是基于光电流 改善的动力学研究, 而就表面修饰对光电压影响的机理 研究较少 ${ }^{[1,18 ~ 20]}$, 电池性能的进一步提升需要全面、系 统的理解表面修饰对电池内微观动力学过程的影响机 制. 本文通过对 $\mathrm{TiO}_{2}$ 薄膜不同时间的电沉积表面修饰, 借助强度调制光电流谱(IMPS)/强度调制光电压谱 (IMVS)等探测技术的动力学表征, 细致研究了表面修 饰后染料敏化太阳电池微观性能的变化机制. 该工作深 化了 DSC 机理研究, 可以为电池性能的进一步改善提 供理论支持.

\section{2 结果与讨论}

在外加电势作用下, $\mathrm{TiCl}_{3}$ 电沉积溶液中的 $\mathrm{Ti}^{3+}$ 及 $\mathrm{OH}^{-}$离子定向移动, 在 $\mathrm{TiO}_{2}$ 薄膜及裸露导电玻璃表面 发生水解反应, 经高温烧结后在表面形成新的 $\mathrm{TiO}_{2}$ 小 颗粒层 ${ }^{[14]}$. 整个反应过程可以用如下方程式来表示 ${ }^{[21]}$ :

$$
\begin{aligned}
& \mathrm{Ti}^{3+}(\mathrm{aq})+\mathrm{H}_{2} \mathrm{O} \longleftrightarrow \mathrm{TiOH}^{2+}+\mathrm{H}^{+} \\
& \mathrm{TiOH}^{2+} \stackrel{-\mathrm{e}}{\longrightarrow} \mathrm{Ti}(\mathrm{IV}) \text { 聚合物 } \frac{450{ }^{\circ} \mathrm{C}}{-\mathrm{H}_{2} \mathrm{O}} \mathrm{TiO}_{2}
\end{aligned}
$$

研究报道 ${ }^{[22]}$ 新生成的 $\mathrm{TiO}_{2}$ 小颗粒层比普通薄膜致 密, 且 $\mathrm{Ti}^{3+}$ 缺陷态的含量有所降低 ${ }^{[12,13]}$. $\mathrm{TiO}_{2} /$ 电解液界 面积累的化学电容可表述为:

$$
C=\mathrm{e}^{2} \frac{\partial n}{\partial E} \approx \mathrm{e}^{2} g(E)
$$

其中, $n$ 为电子浓度 $\left(n=n_{\mathrm{cb}}+n_{\mathrm{ss}}, n_{\mathrm{cb}}\right.$ 和 $n_{\mathrm{ss}}$ 分别为导带电 子浓度和陷阱态电子浓度), $g(E)$ 为陷阱态密度能级分布 函数. 一般情况下 $\left(E_{c}-{ }_{n} E_{\mathrm{F}}>>k_{\mathrm{B}} T\right) n_{\mathrm{ss}}$ 要远大于 $n_{\mathrm{cb}}$, 所 以常用不同电势下的化学电容来表征陷阱态的能级分 布情况. 图 1 为不同沉积时间电池不同偏压下的电容分 布，电容与偏压对应关系为:

$$
C=C_{\mathrm{a}} \exp \left[-\alpha \mathrm{e} V / k_{\mathrm{B}} T\right]+C_{\mathrm{b}}
$$

式中, $C_{\mathrm{a}}, \mathrm{e}, V$ 和 $C_{\mathrm{b}}$ 分别为电容前置因子、电位电荷电 量、外加偏压和电容后置因子, $\alpha$ 表示导带下陷阱态的指 数分布 $(\alpha \leqslant 1)$. 可以看出, 随着沉积时间延长分布系数 $\alpha$ 依次为 $0.19,0.21,0.23$ 和 0.25 , 陷阱态分布变宽(靠近 导带边 $)^{[23]}$. 电沉积水解产生的小颗粒使薄膜质量增加,

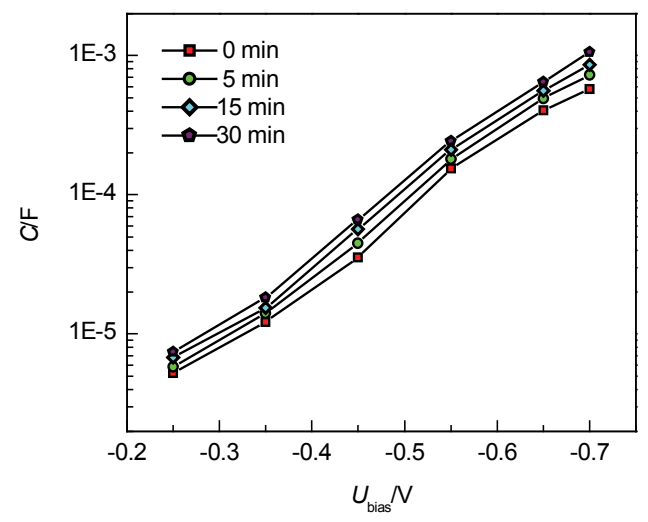

图 1 不同沉积时间电池 $\mathrm{TiO}_{2}$ /电解液界面电容随外加偏压的变化(由 EIS 得到)

Figure 1 Results from the impedance data for the distribution of capacitance for DSCs with different electrodeposition time 
在浅能级引入了大量陷阱态, 以致电势较高时电沉积电 池的化学电容大幅增加 ${ }^{[15]}$.

对于微观动力学过程的研究, 常用电子传输时间 $\tau_{\mathrm{d}}$ (电子从注入 $\mathrm{TiO}_{2}$ 导带至传输至导电玻璃基底收集所 需的时间)表征电子的膜内传输过程, 用电子寿命 $\tau_{\mathrm{n}}$ 表 征复合反应的快慢(电子从注入 $\mathrm{TiO}_{2}$ 导带到被 $\mathrm{I}_{3}^{-}$复合 所需的时间 $)^{[24]}$. 图 2 给出了不同沉积时间电池电子传 输时间及电子寿命随光强的变化. 图 $2 \mathrm{a}$ 可以看出, 电沉 积对 $\mathrm{TiO}_{2}$ 薄膜的表面修饰有效加快了膜内电子传输过 程 ${ }^{[13]}$. 但沉积 $30 \mathrm{~min}$ 电池和沉积 $15 \mathrm{~min}$ 电池的 $\tau_{\mathrm{d}}$ 无明 显差别, 这可能是由于较长时间的沉积易导致小颗粒层 龟裂甚至脱落 ${ }^{[25]}$, 影响了对颗粒间接触的进一步改善 所致. 研究指出小颗粒层可以有效减缓 $\mathrm{TiO}_{2}$ /电解液界 面光生电子与电解液中 $\mathrm{I}_{3}^{-}$的复合速率, 降低内部复 合 ${ }^{[15]}$. 图 $2 \mathrm{~b}$ 为不同沉积时间电池电子寿命 $\tau_{\mathrm{n}}$ 随光强的 变化, $\tau_{\mathrm{n}}$ 对光强的依赖程度可由 $\tau_{\mathrm{n}} \propto \Gamma^{A}$ 表述, $A$ 表示 $\tau_{\mathrm{n}}$ 随 光强变化的快慢 ${ }^{[24,26]}$. 可以看出, 不同沉积时间电池 $\tau_{\mathrm{n}}$ 随光强的变化均比未处理电池要快 $(A=0.41,0.45,0.53$ 和 0.54). 光强较低时电子寿命差别明显, 电沉积处理有 效减缓了复合反应速率; 随着光强增加, 电子费米能级
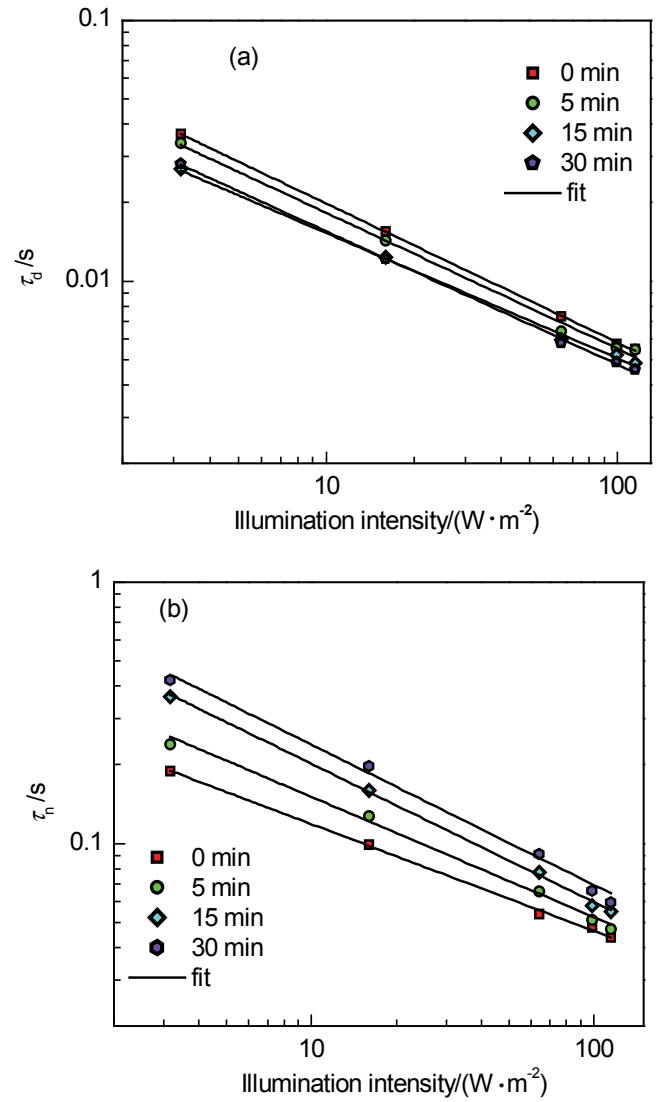

图 2 不同沉积时间电池(a)电子传输时间及(b)电子寿命随光强的变 化

Figure 2 Comparison of (a) electron transport time and (b) electron lifetime constants as a function of light intensities for DSCs with different electrodeposition time
上移，复合的驱动力也随之增加，表面修饰对复合的抑 制作用逐渐减弱, 光强较高时电子寿命的差别明显缩小, 小颗粒层的作用(包括裸露的 TCO 表面)受光强变化影 响显著.

光生电子能否在被复合前注入 $\mathrm{TiO}_{2}$ 膜并传输至导 电玻璃基底收集, 是影响 DSC 光电转换效率的重要因 素之一. 因此, 光生电子复合与传输过程之间存在着竞 争, 可用收集效率 $\eta_{\mathrm{c}}$ 来描述 ${ }^{[9,20]}$

$$
\eta_{\mathrm{c}}=1-\frac{\tau_{\mathrm{d}}}{\tau_{\mathrm{n}}}
$$

可以看出， $\tau_{\mathrm{d}} / \tau_{\mathrm{n}}$ 值越小越利于电子的收集. 图 3 给 出了不同沉积时间电池的电子收集效率随光强变化，随 沉积时间延长, $\eta_{\mathrm{c}}$ 逐渐增大，电沉积对 $\mathrm{TiO}_{2}$ 薄膜的表面 修饰有效改善了膜内电子收集过程.

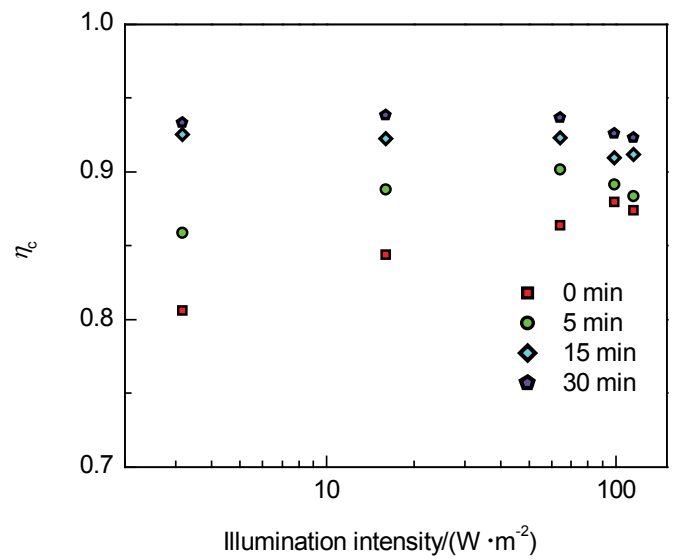

图 3 不同沉积时间电池的电子收集效率随光强变化

Figure 3 Dependence of electron collection efficiency on light intensities for DSCs with different electrodeposition time

光照情况下电子积累在 $\mathrm{TiO}_{2}$ 薄膜中来维持一定的 光电压, 研究发现, DSC 光电压的变化主要受导带边位 置及复合变化决定，即：

$$
\Delta V_{\mathrm{oc}}=\Delta V_{\mathrm{c}}+\Delta V_{\mathrm{F}}
$$

其中, $\Delta V_{\mathrm{c}}$ 和 $\Delta V_{\mathrm{F}}$ 分别为带边移动及内部复合引起的电压 变化 ${ }^{[27,28]}$. 相同电量 $Q\left(J_{\mathrm{sc}} \cdot \tau_{\mathrm{n}}\right)$ 情况下, 导带与电子准费 米能级差值恒定(电解液氧化还原电势保持不变), 导带 正移, 准费米能级也随之移动导致开路电压减小; 导带 负移, 准费米能级也随之移动导致开路电压增大. 因此, 由不同电量 $Q$ 下的 $V_{\mathrm{oc}}$ 可以得到薄膜带边变化情况, 如 图 4 所示. $V_{\mathrm{oc}}$ 与 $\ln (Q)$ 可以线性的表示为 ${ }^{[27]}$

$$
V_{\mathrm{oc}}=V_{\mathrm{c}}+m_{\mathrm{c}} \ln (Q)
$$

式中, $V_{\mathrm{c}}$ 为常数, $m_{\mathrm{c}}$ 为斜率, 即 $m_{\mathrm{c}}=\mathrm{d} V_{\mathrm{oc}} / \mathrm{d} \ln (Q)$. 图 4 可 看出, 随着电沉积时间延长, 薄膜导带边逐渐正移, 沉 积 $30 \mathrm{~min}$ 电池与未处理电池相比导带边降低了约 94 $\mathrm{mV}$, 与 $\mathrm{O}^{\prime}$ Regan 等 ${ }^{[29]}$ 报道结果一致. O'Regan 将这种变 化解释为 $\mathrm{TiO}_{2}$ 薄膜经表面修饰后表面电荷增多及陷阱 态分布变化引起. 导带边的逐渐正移，在改善电子注入 
动力的同时, 也会使 $V_{\mathrm{oc}}$ 有所下降.

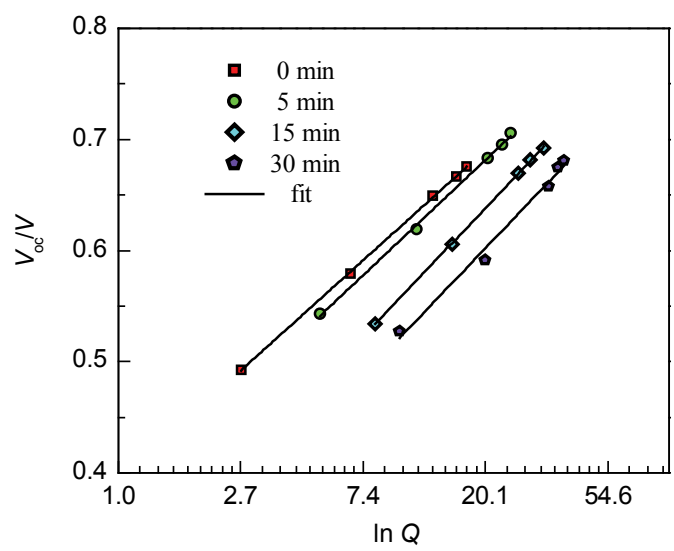

图 4 不同沉积时间电池 $V_{\mathrm{oc}}$ 随 $\ln Q$ 的变化

Figure $4 V_{\text {oc }}$ as a function of $\ln Q$ for DSCs with different electrodeposition time

在 AM1.5 $100 \mathrm{~mW} \cdot \mathrm{cm}^{-2}$ 标准光照条件下, 不同沉 积时间电池 $I-V$ 性能如图 5 所示, 对应电池性能数据见 表 1. 可以看出, 随沉积时间延长 $J_{\mathrm{sc}}$ 逐渐升高, 而 $V_{\mathrm{oc}}$ 却 有逐渐下降的趋势. 与未处理电池相比, $5 \mathrm{~min}$ 电池 $V_{\mathrm{oc}}$ 达最高, $30 \mathrm{~min}$ 电池 $V_{\mathrm{oc}}$ 下降明显. 受带边移动及复合变 化的影响, $5 \mathrm{~min}$ 电池虽然导带正移使 $V_{\mathrm{oc}}$ 略有下降, 但 对复合的抑制却使 $V_{\mathrm{oc}}$ 有所提升, 开路电压对比得出, $-\Delta V_{\mathrm{c}}<\Delta V_{\mathrm{F}}$. 同样, 对于 $30 \mathrm{~min}$ 电池, $-\Delta V_{\mathrm{c}}>\Delta V_{\mathrm{F}}$, 虽 然沉积有效抑制了电子复合, 但导带边的大幅正移使 $V_{\mathrm{oc}}$ 有所下降. 最终, 随着沉积时间延长, $J_{\mathrm{sc}}$ 的逐渐升高 和 $V_{\mathrm{oc}}$ 的逐渐降低使电池效率在沉积时间 $15 \mathrm{~min}$ 时达最 佳.

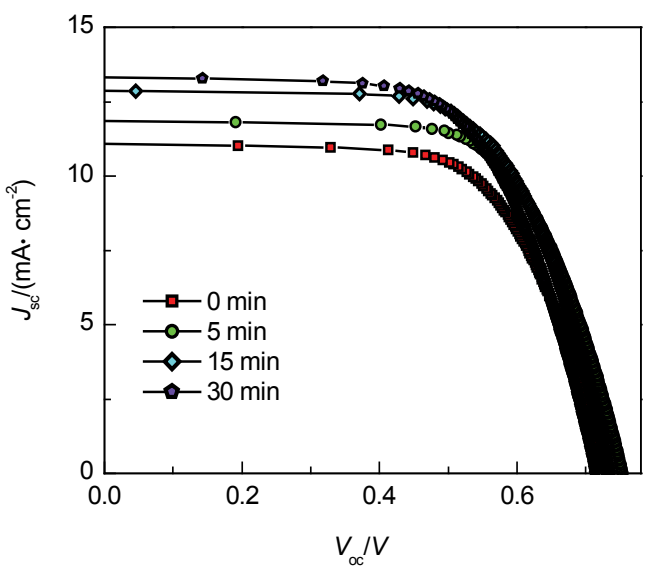

图 5 不同沉积时间电池 AM1.5 $100 \mathrm{~mW} \cdot \mathrm{cm}^{-2}$ 下的 $I-V$ 性能

Figure 5 Photovoltaic performances of DSCs with different electrodeposition time

DSC 的光电转换效率主要由电子产生、注入、收集 过程决定, 可表述为 ${ }^{[30]}$

$\operatorname{IPCE}(\lambda)=\operatorname{LHE}(\lambda) \varphi_{\text {inj }} \eta_{\mathrm{c}}$
表 1 不同沉积时间电池 AM1.5 $100 \mathrm{~mW} \cdot \mathrm{cm}^{-2}$ 下的 $I-V$ 性能

Table 1 Photovoltaic performances of DSCs with different electrodeposition time

\begin{tabular}{rcccc}
\hline Cell & $V_{\text {oc }}$ & $J_{\text {sc }}$ & $F F$ & $\eta$ \\
\hline $0 \mathrm{~min}$ & 0.730 & 11.11 & 0.66 & 5.35 \\
$5 \mathrm{~min}$ & 0.753 & 11.87 & 0.67 & 5.99 \\
$15 \mathrm{~min}$ & 0.733 & 12.85 & 0.66 & 6.22 \\
$30 \mathrm{~min}$ & 0.714 & 13.27 & 0.64 & 6.06 \\
\hline
\end{tabular}

其中, $\operatorname{LHE}(\lambda)$ 为光吸收效率, 与入射光波长及染料吸附 量和染料消光系数有关; $\varphi_{\text {inj }}$ 为光生电子注入效率，与染 料激发态能级及薄膜导带边位置有关; $\eta_{\mathrm{c}}$ 为电子收集效 率, 反映了薄膜对电子的收集能力, 受 $\tau_{\mathrm{d}}, \tau_{\mathrm{n}}$ 大小决定.

图 6 给出了不同沉积时间电池 IPCE 的变化, 可以 看出, 随着沉积时间延长, 电池 IPCE 逐渐增大, 与 $J_{\mathrm{sc}}$ 变化一致. 电沉积小颗粒层在改善收集过程的同时，导 带边的逐渐正移也扩大了染料激发态与薄膜导带间的 能级差，使得光生电子注入动力随沉积时间延长逐渐加 大. 由此可知电沉积对 IPCE 的提升是由注入和收集过 程共同改善引起的.

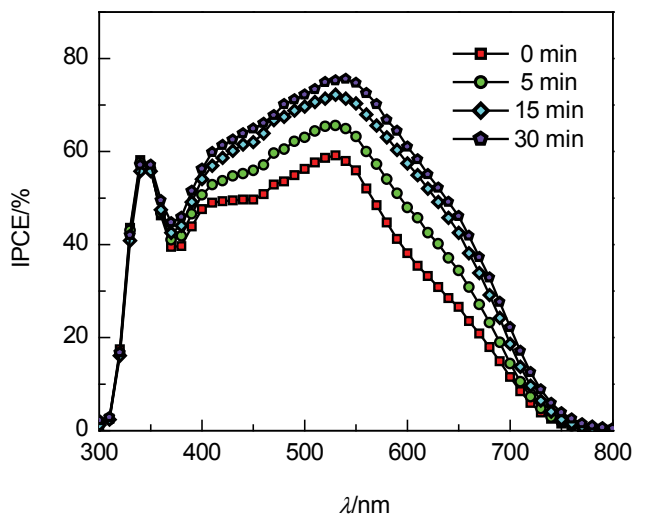

图 6 不同沉积时间电池的 IPCE

Figure 6 The variation of IPCE for DSCs with different electrodeposition time

\section{3 结论}

本文通过染料敏化太阳电池 $\mathrm{TiO}_{2}$ 薄膜不同时间的 电沉积表面修饰后，对微观性能变化机制的研究发现， 电沉积水解的小颗粒层在改善电子注入及收集过程的 同时，也有效抑制了内部电子复合. DSC 开路电压变化 受带边移动及内部复合变化影响: 不同时间表面修饰对 复合的抑制作用使 $V_{\mathrm{oc}}$ 得到不同程度提升; 但是，随着 沉积时间延长, 表面电荷的增多使 $\mathrm{TiO}_{2}$ 薄膜带边逐渐 正移，导致 $V_{\mathrm{oc}}$ 下降. 在两者共同影响下，较长沉积时间 电池导带边的大幅正移限制了光电压的提升. 因此, 采 用电沉积表面修饰对 DSC 光电流和光电压进行改善时, 需要选取适合的沉积时间. 


\section{4 实验部分}

纳米 $\mathrm{TiO}_{2}$ 浆料、纳米 $\mathrm{TiO}_{2}$ 多孔薄膜、染料以及电 解液的制备详见文献 $[31,32]$. 利用丝网印刷技术在导 电玻璃(TEC-15, LOF)上印刷 $\mathrm{TiO}_{2}$ 浆料, 通过马弗炉于 $510{ }^{\circ} \mathrm{C}$ 烧结 $30 \mathrm{~min}$, 薄膜厚度约 $13 \mu \mathrm{m}$, 有效面积为 0.25 $\mathrm{cm}^{2}$ (为保证沉积效果, 多孔薄膜均未加印大颗粒散射 层). 电沉积法表面修饰薄膜采用三电极体系: 工作电 极为 $\mathrm{TiO}_{2}$ 多孔薄膜、对电极为 $\mathrm{Pt}$ 片、参比电极为 SCE. 电镀液的配制将 $0.1 \mathrm{~g} \mathrm{NaCl}$ 加入到 $70 \mathrm{~g}$ 高纯水(除氧)中 摚拌, 随后滴入 $3.9 \mathrm{~g}$ 用 $\mathrm{Zn}$ 粒处理数天的 $\mathrm{TiCl}_{3}$ 盐酸溶 液. 将已除氧的饱和 $\mathrm{NaHCO}_{3}$ 溶液加入到 $\mathrm{TiCl}_{3}$ 溶液中, 调整 $\mathrm{pH}$ 值至 2.2 , 在外加恒电势 $0.1 \mathrm{~V}$ vs SCE 下进行电 沉积操作 ${ }^{[1,21]}$. 修饰电极经再次烧结后, 室温下在 N719 染料的乙醇溶液中浸泡 $12 \mathrm{~h}$ 取出. Pt 对电极的制备采用 将 $\mathrm{H}_{2} \mathrm{PtCl}_{6}$ 溶液喷涂到透明导电玻璃上, 通过马弗炉于 $410{ }^{\circ} \mathrm{C}$ 烧结 $20 \mathrm{~min}$ 得到. 两个电极热压密封后, 通过对 电极的小孔注入电解质溶液, 再对小孔进行密封制成电 池.

电化学阻抗谱(EIS)通过德国 Zahner 公司生产的 IM6ex 型电化学工作站测量, 调制振幅为 $5 \mathrm{mV}$, 频率范 围为 $1000 \mathrm{kHz} \sim 0.1 \mathrm{~Hz}$. 实验数据利用 Zview 软件拟合. IMPS/IMVS 的测试则由可控强度调制光谱仪(CIMPS, Zahner, 德国)完成: 光源为 Expot 驱动的波长 $610 \mathrm{~nm}$ LED, 调制光强振幅约为背景光强的 $10 \%$, 测试频率范 围 $3 \mathrm{kHz} \sim 0.1 \mathrm{~Hz}$.

DSC $I-V$ 性能的测试利用太阳模拟器(solar AAA simulator, oriel USA)和美国 Keithley 2420 型数字源表, 通过 Testpoint 伏安特性测试软件自动完成. IPCE 测试 借助 Newport 公司的 IPCE 测试系统, 所有的部件和整 个测量过程由 Oriel® Tracq Basic V5.0 软件控制自动进 行.

\section{References}

[1] Kou, D. X.; Liu, W. Q.; Hu, L. H.; Huang, Y.; Dai, S. Y.; Jiang, N. Q. Acta Phys. Sinica 2010, 59, 5857. (寇东星, 刘伟庆, 胡林华, 黄 阳，戴松元，姜年权，物理学报, 2010, 59, 5857.)

[2] Li, J.; Kong, F. T.; Zhang, C. N.; Liu, W. Q.; Dai, S. Y. Acta Chim. Sinica 2010, 68, 1357. (李洁, 孔凡太, 张昌能, 刘伟庆, 戴松元, 化学学报, 2010, 68, 1357.)

[3] Huang, X. W.; Deng, J. Y.; Xu, L.; Shen, P.; Zhao, B.; Tan, S. T. Acta Chim. Sinica 2012, 70, 1604. (黄先威, 邓继勇, 许律, 沈平, 赵 斌, 谭松庭, 化学学报, 2012, 70, 1604.)
[4] Oregan, B.; Gratzel, M. Nature 1991, 353, 737.

[5] Yella, A.; Lee, H. W.; Tsao, H. N.; Yi, C. Y.; Chandiran, A. K.; Nazeeruddin, M. K.; Diau, E. W. G.; Yeh, C. Y.; Zakeeruddin, S. M.; Gratzel, M. Science 2011, 334, 629.

[6] Liu, W. Q.; Kou, D. X.; Hu, L. H.; Huang, Y.; Jiang, N. Q.; Dai, S. Y. Acta Phys. Sinica 2010, 59, 5141. (刘伟庆，寇东星，胡林华，黄 阳，姜年权，戴松元，物理学报, 2010, 59, 5141.)

[7] Huang, Y.; Dai, S. Y.; Chen, S. H.; Hu, L. H.; Kong, F. T.; Kou, D. X.; Jiang, N. Q. Acta Phys. Sinica 2010, 59, 643. (黄阳, 戴松元, 陈双宏, 胡林华, 孔凡太, 寇东星, 姜年权, 物理学报, 2010, 59, 643.)

[8] Peter, L. M. Phys. Chem. Chem. Phys. 2007, 9, 2630.

[9] Park, J. H.; Kim, J. H.; Choi, C. J.; Kim, H.; Ahn, K. S. Molecular Crystals and Liquid Crystals 2012, 567, 19.

[10] Nazeeruddin, M. K.; Kay, A.; Rodicio, I.; Humphrybaker, R.; Muller, E.; Liska, P.; Vlachopoulos, N.; Gratzel, M. J. Am. Chem. Soc. 1993, 115,6382 .

[11] Hart, J. N.; Menzies, D.; Cheng, Y. B.; Simon, G. P.; Spiccia, L. Comptes Rendus Chimie 2006, 9, 622.

[12] Lee, C.; Lee, G. W.; Kang, W.; Lee, D. K.; Ko, M. J.; Kim, K.; Park, N. G. Bull. Korean Chem. Soc. 2010, 31, 3093.

[13] Lee, S. W.; Ahn, K. S. J. Phys. Chem. C 2012, 116, 21285.

[14] Lee, S.; Jun, Y.; Kim, K. J.; Kim, D. Sol. Energy Mater. Sol. Cells 2001, 65, 193.

[15] Shi, J. F.; Liang, J.; Peng, S. J.; Xu, W.; Pei, J.; Chen, J. Solid State Sci. 2009, 11, 433.

[16] Park, J. H.; Kim, J. Y.; Kim, J. H.; Choi, C. J.; Kim, H.; Sung, Y. E.; Ahn, K. S. J. Power Sources 2011, 196, 8904.

[17] Xu, W. W.; Dai, S. Y.; Fang, X. Q.; Hu, L. H.; Kong, F. T.; Pan, X.; Wang, K. J. Acta Phys. Sinica 2005, 54, 5943. (徐炜炜, 戴松元, 方霞琴, 胡林华, 孔凡太，潘旭，王孔嘉，物理学报，2005, 54, 5943.)

[18] Hagfeldt, A.; Gratzel, M. Chem. Rev. 1995, 95, 49.

[19] Hagfeldt, A.; Gratzel, M. Acc. Chem. Res. 2000, 33, 269.

[20] Zhu, K.; Kopidakis, N.; Neale, N. R.; van de Lagemaat, J.; Frank, A. J. J. Phys. Chem. B 2006, 110, 25174.

[21] Kavan, L.; Oregan, B.; Kay, A.; Gratzel, M. J. Electroanal. Chem. 1993, 346, 291.

[22] Wang, M.; Chen, P.; Humphry-Baker, R.; Zakeeruddin, S. M.; Gratzel, M. ChemPhysChem 2009, 10, 290.

[23] Wang, Q.; Zhang, Z.; Zakeeruddin, S. M.; Gratzel, M. J. Phys. Chem. C 2008, 112, 7084 .

[24] Fisher, A. C.; Peter, L. M.; Ponomarev, E. A.; Walker, A. B.; Wijayantha, K. G. U. J. Phys. Chem. B 2000, 104, 949.

[25] Karuppuchamy, S.; Nonomura, K.; Yoshida, T.; Sugiura, T.; Minoura, H. Solid State Ionics 2002, 151, 19.

[26] Duffy, N. W.; Peter, L. M.; Wijayantha, K. G. U. Electrochem. Commun. 2000, 2, 262.

[27] Schlichthorl, G.; Huang, S. Y.; Sprague, J.; Frank, A. J. J. Phys. Chem. B 1997, 101, 8141.

[28] Liu, W. Q.; Kou, D. X.; Cai, M. L.; Hu, L. H.; Sheng, J.; Tian, H. J.; Jiang, N. Q.; Dai, S. Y. J. Phys. Chem. C 2010, 114, 9965.

[29] O'Regan, B. C.; Durrant, J. R.; Sommeling, P. M.; Bakker, N. J. J. Phys. Chem. C 2007, 111, 14001.

[30] Zhu, K.; Neale, N. R.; Miedaner, A.; Frank, A. J. Nano Lett. 2007, 7, 69.

[31] Guo, L.; Pan, X.; Wang, M.; Kou, D. X.; Cai, M.; Zhang, C. N.; Dai, S. Y. Acta Chim. Sinica 2011, 69, 767. (郭否, 潘旭, 王猛, 寇东星, 蔡墨朗, 张昌能, 戴松元, 化学学报, 2011, 69, 767.)

[32] Hu, L. H.; Dai, S. Y.; Weng, J.; Xiao, S. F.; Sui, Y. F.; Huang, Y.; Chen, S. H.; Kong, F. T.; Pan, X.; Liang, L. Y.; Wang, K. J. J. Phys. Chem. B 2007, 111, 358 . 